\title{
Editorial
}

\section{Rudolf Fisch}

Spätestens seit der Jahrtausendwende wird das Thema „computergestützte Kommunikation in formalen Organisationen“, ihre Chancen und ihre Probleme, in ManagementMagazinen und in der Management-Ratgeberliteratur diskutiert. In der Regel werden vor allem die Chancen beschrieben und dabei mehr oder weniger offen für diese Arbeitsform geworben, mit unterschiedlichsten Argumenten. Mögliche Probleme dieser Arbeitsform oder kritische Berichte von Nutzern werden an solchen Stellen kaum dargestellt.

Großes Interesse findet das Thema computerunterstützte kooperative Arbeit in virtuellen Gruppen wie auch in realen Arbeitsgruppen. Notwendig für diese relativ neue Arbeitsform war die Entwicklung einschlägiger Software. Zwei Beispiele zur Illustration: Die Software „Lotus Notes“, wurde vor mehr als zwanzig Jahre speziell entwickelt für kooperative Arbeit an einer Aufgabe zu verschiedenen Zeiten an verschiedenen Orten. Die Softwarefirma CAS (Karlsruhe) bietet seit den 90er Jahren des zurückliegenden Jahrhunderts Software zur Koordination der Arbeit in (Projekt)Gruppen an, aktuell als „CAS genesisWorld“. Heute werden für die computergestützte Arbeit in Gruppen vorzugsweise Internet-Plattformen verschiedenster Art genutzt. Ihre Anwendung ist nicht auf die Arbeitswelt beschränkt. Derartige Software und Plattformen finden in Lernkontexten wie Schulen, Hochschulen und Universitäten und vor allem in der berufsbegleitenden Fortbildung breite Verwendung.

Eine besondere Herausforderung technischer wie menschlicher Art stellt die Arbeit in virtuellen Arbeitsgruppen dar, in denen ortsverteilt, auch über Kontinente hinweg, an einer Aufgabe gearbeitet wird, zum Beispiel an der Entwicklung von Software oder an einer technischen Entwicklungsaufgabe zum Beispiel im Automobilbau oder bei der Entwicklung pharmazeutischer Präparate.

Mit dem Aufkommen der Tablet-Computer scheint noch einmal eine ganz neue Ära angebrochen zu sein. Tablet-Computer erlauben weitergehende Informations- und Kommunikationsformen in Organisationen als bisher. Zum Beispiel: Teilnehmerinnen und Teilnehmer an einer Arbeitssitzung in einem Unternehmen können, versehen mit den entsprechenden Zugriffsrechten, anlässlich der Erörterung kritischer Fragen sofort auf die Datenbank ihrer Firma zurückgreifen. Sitzungsteilnehmende beschaffen sich auf

Online publiziert: 05.11 .2013

(C) Springer Fachmedien Wiesbaden 2013

Prof. Dr. R. Fisch $(\bowtie)$

Konstanz, Deutschland

E-Mail: fisch@dhv-speyer.de 
diese Weise zu einem Sachverhalt aktuell aufbereitete Informationen. Damit können sie daten- und faktengestützte Informationen unmittelbar in die Erörterung einbringen. Früher hätte man zur Klärung der Fragen einen neuen Sitzungstermin anberaumen müssen. Oder Gemeinden senden ihren Gemeinderatsmitgliedern zur Vorbereitung einer Sitzung keine Pakete mit Sitzungsunterlagen mehr, sondern statten sie mit Tablet-Computern aus, damit Protokolle und Vorlagen von einer geeigneten Plattform abgerufen werden können. Papiere werden nicht mehr versandt.

In Büros gehen mit dem Gebrauch solcher neuer Informationstechniken andere Führungsformen einher, zum Beispiel: Über einen Arbeitseinheits-Blog informiert der oder die ortsabwesende Vorgesetzte über die nächsten Gesprächskontakte in den kommenden Tagen. Aufmerksame Mitarbeiterinnen und Mitarbeiter können von sich aus bedeutsame Informationen und Unterlagen für die Gesprächsvorbereitung aufbereiten und übermitteln, ohne dass von Vorgesetztenseite extra dazu nachgefragt werden müsste. Und die Mitarbeitenden sind durch diese Arbeitsform zugleich über bedeutsame Geschäftsprozesse informiert.

Und eine dritte, wahrscheinlich folgenreiche Innovation sei erwähnt, die allerdings erst im Entstehen begriffen ist: eGovernment. Es umfasst die Aufgabe, Regieren und Verwalten und was auf allen Ebenen dazu gehört, im Internet transparent darzustellen und damit unter anderem die Grundlage für breite Bürgerbeteiligung auf informationeller Basis zu schaffen.

Bei alle dem ist zu bedenken: Menschen sind verschieden! Sie denken verschieden, haben unterschiedliche informationelle Bedürfnisse, unterschiedlich ausgeprägte soziale Motive bei der Zusammenarbeit, verfügen über unterschiedliche, auch kulturell vermittelte Interaktionsstile bei der Zusammenarbeit in Arbeitseinheiten, wissen unterschiedlich viel über die hier angesprochenen besonderen Arbeitsformen, kennen sich unterschiedlich mit den Geräten, der Software und den technischen Bedingungen aus, wie zum Beispiel mit den Besonderheiten einer Videokonferenz und so fort. Doch: die wie auch immer einmal gewählte Technik oder Software für die Kommunikation ist einheitlich, folgt den Notwendigkeiten und der Logik der Programmierung in der Datenverarbeitung oder der von Maschinen. Die Bedienung der Geräte ist weniger auf die Unterschiedlichkeit von Menschen und deren Kommunikationspräferenzen hin angelegt. Daher liegt die Frage nahe: Wie kommen die Nutzer mit den neuen informations- und kommunikationstechnischen Instrumenten zurecht? Was fördert die Arbeit, was behindert sie? Wenn es Probleme geben sollte: Wie könnten allfällig mögliche Lösungen aussehen?

Das vorliegende Themenheft gibt einige Einblicke in aktuelle sozialwissenschaftliche Untersuchungen zu diesen Fragen. Zum einen geht es um Chancen und Folgen der kommunikationstechnischen Neuerungen in der Arbeitswelt, zum anderen um besondere Formen des Lernens in der formalen (Weiter)Bildung. Die beiden Bereiche sind Schwerpunkte der Anwendung der neuen Techniken. Zugleich erkennt man in den vorgelegten Studien die nicht unerhebliche Diskrepanz zwischen den Werbeversprechen der Computer- und Softwarebranche und dem, was aufseiten der Nutzer an Chancen, Möglichkeiten und Bewertungen gesehen wird. Angesichts der weiter fortschreitenden tiefgreifenden Änderungen im Bereich Führung und Zusammenarbeit durch die neuen Techniken darf man gespannt sein, was in Zukunft auf diesem Gebiet an einschlägigen Forschungsarbeiten entstehen wird. 